\title{
PROGRESS OF THE LONGITUDINAL FEEDBACK SYSTEM AND ASSOCIATED BEAM OBSERVATIONS IN TLS
}

\author{
W. K. Lau, L. H. Chang, P. J. Chou, K. T. Hsu, C. H. Kuo, M. H. Wang, T. T. Yang, M. S. Yeh, \\ SRRC, Hsinchu, Taiwan
}

\begin{abstract}
A digital feedback control system against longitudinal coupled-bunch instability is being developed for the Taiwan Light Source (TLS). This system is designed to stabilize bunch-phase oscillation of each of the 200 electron bunches in the TLS storage ring, and it operates at a bunch crossing frequency of $500 \mathrm{MHz}$. Two innovative aspects of this system are (1) the special design of the digital signal processing (DSP) electronics, which run at a peak data rate of 500 Mbytes per second, and (2) the compact broadband longitudinal kicker for bunchphase correction. Recent progress of this work and preliminary results of associated observations of beam longitudinal-dynamics will also be reported.
\end{abstract}

\section{INTRODUCTION}

Longitudinal coupled-bunch instability (LCBI) that occurred in the TLS storage ring electron beam is believed to be driven by cavity high order modes (HOMs). Since LCBI is the main obstacle in preserving the natural emittance at higher beam current. They broaden the spectra of undulators and which cause slow fluctuations of the photon beam intensity. A digital feedback control system is designed to stabilize dipole-mode LCBI in a bunch-by-bunch manner. Design of such system has been discussed elsewhere [1]. However, specific constraints have to be considered when such system is designed to operate in the TLS storage ring. First, maximum longitudinal dimension is limited to $230 \mathrm{~mm}$ (including the transitions from round to elliptical beam pipes) which is relatively short in comparison with other kicker structure design. Second, the expectations from the users require that the system have to be operational within a short period. In order to reduce system development time, commercially available embedded DSP modules were chosen as the core of signal processing electronics - a DSP array performs parallel digital signal filtering. A unique signal conversion plus data-transfer unit was developed as the interface between the fast changing analog signals and the DSP array (in which DSP chips run at a slower processing rate). A compact longitudinal kicker with 250 $\mathrm{MHz}$ bandwidth (FWHM) operates at a center frequency of $1125 \mathrm{MHz}$ to provide energy kicks to the bunches. This structure is equipped with magnetic loops which permit rf power to be coupled out from the structure (through high power, coaxial, rf feedthroughs) in order to "de-Q" the acceleration mode for wide-bandwidth and to damp high- order modes. Also, rf power can be coupled into the kicker through symmetrically placed ports for kicker voltage enhancement. This paper reports the progress of the development of such system. During the construction stage, longitudinal dynamics has been observed by using part of the system. For examples, the bunch phase detector is used to measure synchrotron tunes via beam transfer function measurements in the single bunch mode. A preliminary observation of bunch phase oscillations induced by zero-LCBI mode.

\section{SUMMARY OF RECENT PROGRESS}

\subsection{Installation of the Longitudinal Kicker}

The longitudinal kicker described above has recently been installed into the storage ring (Figure 1). It has been used to kick the beam with voltage modulation on the reference signal that drive the 200 watts power amplifier. The power of synchrotron sideband has increased by more than $10 \mathrm{~dB}$ when full power was applied to the kicker. Beam signal picked-up from one of the magnetic loop of the kicker is used to estimate the kicker shunt impedance and bandwidth. At very low beam current, the observed signal power at $1 \mathrm{GHz}$ is measured. The deduced shunt impedance is quite close to its predicted value that is about 100 Ohms. The strength of the picked-up beam signal is maximized within a bandwidth of about $250 \mathrm{MHz}$ as expected.

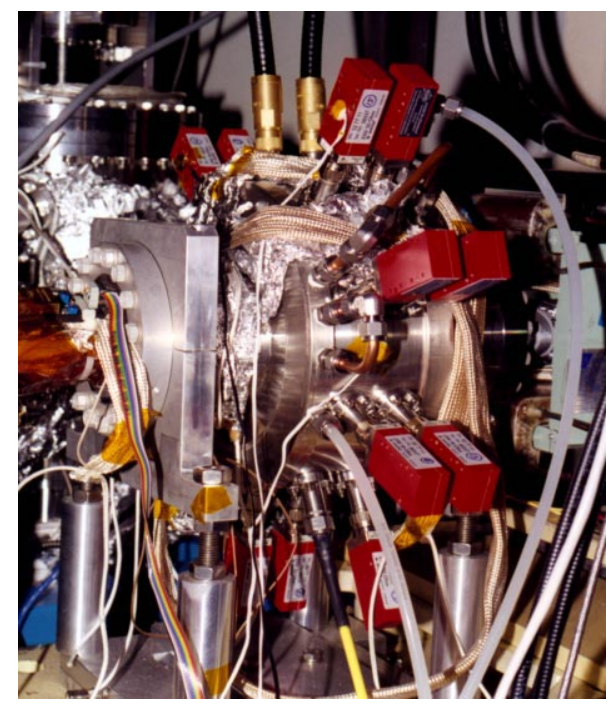

Figure 1: The longitudinal kicker installed in the TLS storage ring 


\subsection{The Bunch Phase Detector and the Wide- band RF System}

The bunch phase detector and the 1.0-1.25 GHz rf system has been built and tested. The detector has been used for beam observation (see Section 3), physically meaningful results has been obtained.

The correction signal from the DAC is used to modulate the $1 \mathrm{GHz}$ carrier signal (locked to the ring master clock) that drive the power amplifier. A double side-band carrier suppressed (DSB-CS) modulator is made for this purpose during initial beam test. A QPSK modulated $1.125 \mathrm{GHz}$ locked source is being built to replace the $1 \mathrm{GHz}$ carrier when it is needed. Now, the system is equipped with a 200 Watts power amplifier with instantaneous bandwidth from 1.0-2.0 GHz.

\subsection{Signal Processing Electronics}

Signal processing electronics is perhaps the most time consuming part in developing the feedback system. Since the $60 \mathrm{MHz}$ C44 DSP chips is chosen as the signal processing element, digitized signal is transfer to the DSP modules via communication ports (modules with even faster DSP chips are now under evaluation). The ADC/DEMUX unit has been built to digitize the analog bunch phase signal into 8 bits data and are distributed to the DSP chips [2]. The DAC/MUX unit is equipped with hold buffer circuitry that holds the digital filter output for eighteen turns. Simulation shows that the system gain is significantly increased with this hold buffer capability [3]. Full speed system can only be possible until the DAC/MUX unit is completed.

\subsection{Feedback with Main Cavity as Kicker}

Single bunch experiment can be performed to test subsystem and parts online in this intermediate stage.

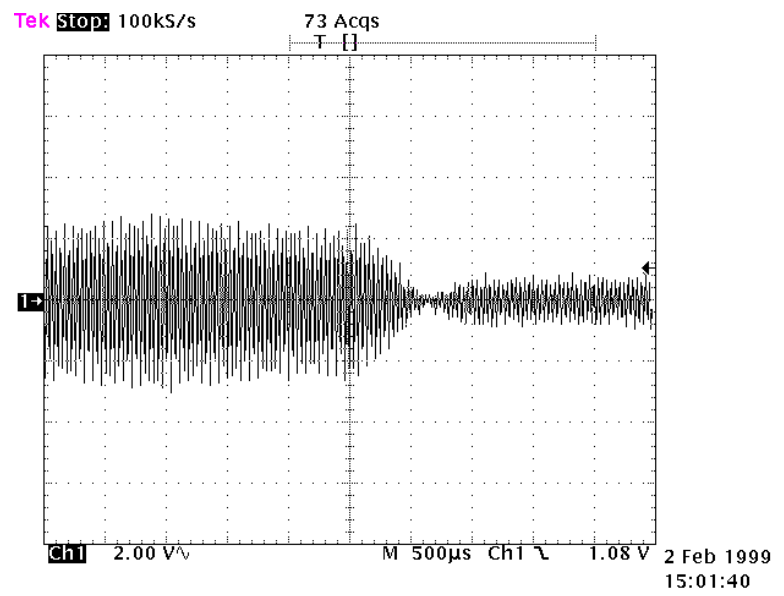

Figure 2: Result of feedback with main cavity as kicker. Showing phase oscillation of a bunch is damped
Figure 2 shows the amplitude of the phase oscillation of a bunch is damped to a small value within a few synchrotron oscillation cycles.

The TLS rf system consists of two independent rf stations. We used one of the cavity as a kicker to drive bunch phase oscillations and with the other one in the feedback loop to damp the oscillation (the compact longitudinal kicker is not available during the experiment). In this experiment, a 4-tap FIR filter is used. However, further optimization in controller design is required. We are now working on single bunch experiment with the installed longitudinal kicker.

\section{PRELIMINARY OBSERVATIONS OF LONGITUDINAL BEAM DYNAMICS}

Since the bunch phase detector, ADC/DEMUX unit and DSP modules are partially installed, some observations on beam longitudinal dynamics are now become possible.

\subsection{Tune Measurement}

Longitudinal beam transfer function can be measured by injecting phase modulation to the main cavity voltage and bunch phase oscillation is detected by the system bunch phase detector. From this measurement, synchrotron tune as a function of gap voltage can be found. It shows good agreement between theoretical and measured data.

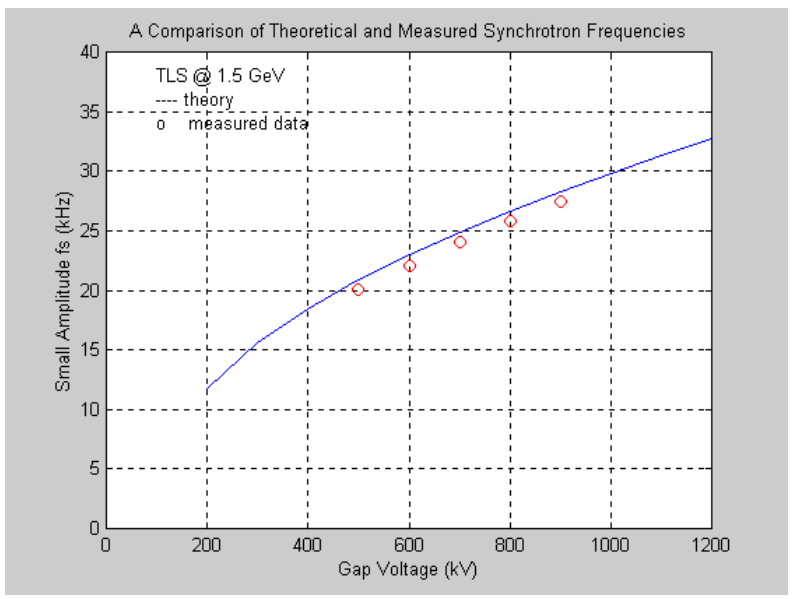

Figure 3: Measured synchrotron tune versus gap voltage in TLS

\subsection{Observation of a Zero-mode LCBI}

Zero-mode LCBI is excited in purpose by de-tuning the main cavities, phase error signals of each bunches are detected and digitized and distributed to the DSP modules by the ADC/DEMUX unit. Data representing 25 equally spaced bunches are collected by one of the DSP. 


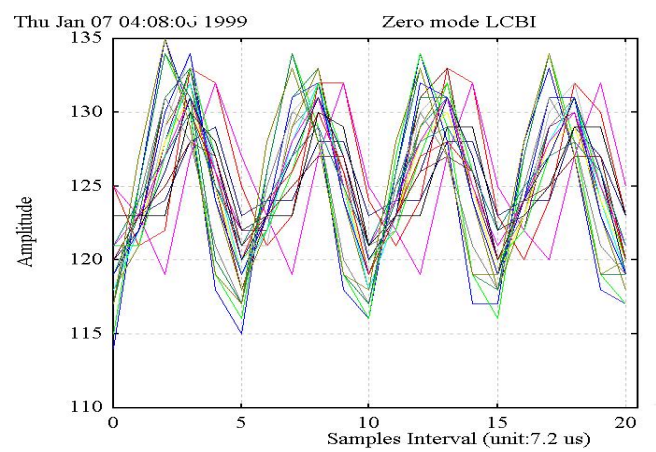

Figure 4: The measured coherent phase oscillation of the bunches is a characteristic of zero-mode LCBI

\section{DISCUSSIONS}

Recent progress of developing a digital longitudinal feedback is reported. The system is partially installed. Beam observation has been made to verify subsystem performance. Since we have a major modification that a hold buffer circuit is included into the DAC/MUX unit, the schedule may be delayed for a few of months. The full speed system test will be performed in near future.

\section{REFERENCES}

[1] W. K. Lau et al., "Development of a Digital Longitudinal Damper for the TLS Storage Ring" Proceedings of the Particle Accelerator Conference 1997.

[2] M. S. Yeh et al., "High Speed Digital Signal Processing Electronics for the TLS Longitudinal Feedback System" these proceedings.

[3] W. K. Lau et al., "Analysis of the TLS Longitudinal Feedback System" these proceedings. 